\title{
Singular Pair Breaking in the Composite Fermi Liquid Description of the Half-Filled Landau Level
}

\author{
N. E. Bonesteel \\ National High Magnetic Field Laboratory and Department of Physics, Florida State University, \\ Tallahassee, FL 32310
}

(April 23, 2018)

\begin{abstract}
Fluctuations of the Chern-Simons gauge field in the composite Fermi liquid description of the half-filled Landau level are pair breaking in all angular momentum channels. For short-range electron-electron interactions these fluctuations are sufficiently strong to drive any $T=0$ pairing transition first order. For Coulomb interactions these fluctuations are weaker and a continuous transition is possible.
\end{abstract}

73.20.Dx,05.30.-d,73.40.Hm

Typeset using REVTEX 
There is strong experimental support [1] for the remarkable hypothesis that the twodimensional electron gas at Landau-level filling fraction $\nu=1 / 2$ can be viewed as a compressible 'metal' with a sharp Fermi surface [2]. The initial formulation of this idea, based on the composite fermion theory of the fractional quantum Hall effect [3], involved representing physical electrons by fermions, referred to in what follows as Chern-Simons (CS) fermions, bound to two quanta of fictitious, or CS, flux [2, [1,5]. If this flux is chosen to point in the direction opposite to that of the physical magnetic field then, at the mean-field level, CS fermions at $\nu=1 / 2$ see zero effective field and form a metallic state with a Fermi surface. Halperin, Lee, and Read (HLR) [2] developed a theoretical description of the resulting 'composite Fermi liquid' (CFL) by studying fluctuations about this mean-field state within the random-phase approximation (RPA).

Unlike the CS theories used to describe incompressible fractional quantum Hall states [5.66], where the presence of an energy gap provides some justification for thinking that fluctuations are under control, the CFL is gapless, the fluctuations are large, and the degree to which the mean-field solution captures the essential physics is unclear. One question with important physical consequences is whether or not the mean-field Fermi surface is stable. Greiter, Wen, and Wilczek (GWW) [7] have shown that the bare 'density-current' interaction between the flux attached to one CS fermion and the current of another CS fermion mediates an attractive pairing interaction in the $p$-wave channel. These authors argued further that the resulting paired state corresponds to the incompressible Pfaffian state originally proposed by Moore and Read [8].

The fractional quantum Hall state at $\nu=5 / 2$ [9] provides experimental motivation for studying the stability of the CFL. Recent exact diagonalization calculations of Morf [10] have shown the for the Coulomb interaction the half-filled first-excited Landau level at $\nu=5 / 2$ is spin polarized, even in the absence of Zeeman coupling. Morf further speculates that the observed collapse of this state in tilted fields [11] is a consequence of the 'hardening' of the short-range part of the electron-electron interaction due to the 'pinching' of the lowest subband wave function of the two dimensional electron gas [12]. If this is the case then 
it is plausible that the incompressible state at small tilt is a Pfaffian, and the tilted field transition is from Pfaffian to CFL. Another scenario, advocated by Rezayi and Haldane [13], is that for a pure system there is no transition - the CFL is always unstable to the Pfaffian - and the observed incompressible-compressible transition occurs when the gap becomes smaller than the characteristic decay width associated with the disorder.

The analysis of GWW favors the latter scenario, that in the absence of disorder the CFL is always unstable to the formation of a paired quantum Hall state. It is the modest purpose of this paper to show that while this may in fact be the case it is not necessarily the case. If one goes beyond GWW and, following [2], computes the effective interaction between CS fermions within the RPA, the current-current interaction mediated by the transverse CS gauge fluctuations is more singular than the density-current interaction considered by GWW and is strongly pair breaking in all angular momentum channels. These fluctuations can, in principle, stabilize the CFL. In what follows it will be assumed, with the usual provisos regarding renormalization of the effective mass, that the HLR approach is qualitatively correct. The relevance of the present work to more recent theoretical formulations of the CFL [14]19] will depend on the extent to which these theories resemble HLR, currently a matter of some controversy [20].

Consider a two dimensional electron gas, realized in the $x y$ plane, in a perpendicular magnetic field $B$ at filling factor $\nu=1 / \tilde{\phi}$, where $\tilde{\phi}$ is an even integer. Taking $\hbar=c=1$ the magnetic field is $B=2 \pi \tilde{\phi} n / e$ where $n$ is the electron density. This system can be described by the Euclidean time action [2] $\mathcal{S}=\int_{0}^{\beta} d \tau \mathcal{L}_{0}(\tau)+\mathcal{S}_{C S}$, where

$$
\mathcal{L}_{0}(\tau)=\int d^{2} r \bar{\psi}\left(\partial_{\tau}-a_{0}-\mu-\frac{1}{2 m}(\nabla-i \mathbf{a}+i e \mathbf{A})^{2}\right) \psi
$$

and

$$
\mathcal{S}_{C S}=\frac{1}{2} \sum_{\mu, \nu} \sum_{\mathbf{q}, n} a_{\mu}\left(\mathbf{q}, \omega_{n}\right) \mathcal{D}_{\mu \nu}^{0}{ }^{-1}(q) a_{\nu}\left(\mathbf{q}, \omega_{n}\right) .
$$

Here $\psi$ is the CS fermion field, $a_{0}$ and $a_{1}\left(\mathbf{q}, \omega_{n}\right)=\hat{\mathbf{z}} \cdot\left(\hat{\mathbf{q}} \times \mathbf{a}\left(\mathbf{q}, \omega_{n}\right)\right)$ are the time and transverse components of the corresponding statistical gauge field, $\omega_{n}=2 n \pi / \beta$ is a bosonic 
Matsubara frequency, $\mathbf{A}(\mathbf{r})=(\hat{\mathbf{z}} \times \mathbf{r}) B / 2$ is the physical vector potential describing the applied magnetic field, $m$ is the band mass of the electrons, and

$$
\mathcal{D}^{0}(q)=\left(\begin{array}{cc}
v(q) & i 2 \pi \tilde{\phi} / q \\
-i 2 \pi \tilde{\phi} / q & 0
\end{array}\right)
$$

is the 'bare' CS propagator where $v(q)$ is the electron-electron interaction. Integrating out $a_{0}$ enforces the constraint $\boldsymbol{\nabla} \times \mathbf{a}=\hat{\mathbf{z}} 2 \pi \tilde{\phi} \bar{\psi} \psi$ so that at the mean-field level $\langle\boldsymbol{\nabla} \times \mathbf{a}\rangle=\hat{\mathbf{z}} 2 \pi \tilde{\phi} n=$ $\hat{\mathbf{z}} e B$. The CS gauge field then exactly cancels the physical magnetic field and the CS fermions form a Fermi liquid with Fermi wave vector $k_{F}=(2 / \tilde{\phi})^{1 / 2} / l_{0}$ where $l_{0}=(1 / e B)^{1 / 2}$ is the magnetic length.

The pairing instability discussed by GWW [7] is due to the bare 'statistical' interaction in the Cooper channel,

$$
V_{10}^{0}\left(\mathbf{k}, \mathbf{k}^{\prime}\right)=\frac{\mathbf{k} \times \hat{\mathbf{q}}}{m} \mathcal{D}_{10}^{0}(q)=i \frac{\mathbf{k} \times \hat{\mathbf{q}}}{m} \frac{2 \pi \tilde{\phi}}{q},
$$

where $\mathbf{q}=\mathbf{k}-\mathbf{k}^{\prime}$. A dimensionless coupling constant characterizing the strength of a given pairing interaction can be obtained by taking the Fermi-surface average in the $l$-wave channel,

$$
\lambda(\omega)=-\frac{m}{2 \pi} \int_{0}^{2 \pi} V\left(k_{F} \hat{\mathbf{x}}, k_{F}(\cos \theta \hat{\mathbf{x}}+\sin \theta \hat{\mathbf{y}}), \omega\right) \exp (i \theta l) d \theta,
$$

where the sign is chosen so that $\lambda$ is positive for an attractive interaction. Because the CS fermions are spinless $l$ must be odd. For $V_{10}^{0}$ there is no frequency dependence and the result of this integral is $\lambda_{10}^{0}=\operatorname{sgn}(l) \pi \tilde{\phi}$ [21]. The interaction is attractive for positive $l$ and repulsive for negative $l$, reflecting the fact that the pairing interaction is not time-reversal symmetric.

The RPA expression for the CS gauge field propagator is obtained by integrating out the CS Fermi fields and expanding the resulting effective action to second order in the CS gauge fields with the result [2]

$$
\mathcal{S}_{R P A}=\frac{1}{2} \sum_{\mu, \nu} \sum_{\mathbf{q}, n} a_{\mu}\left(\mathbf{q}, \omega_{n}\right) \mathcal{D}_{\mu \nu}^{-1}\left(q, \omega_{n}\right) a_{\nu}\left(\mathbf{q}, \omega_{n}\right) .
$$


Here $\mathcal{D}^{-1}=\mathcal{K}^{0}+\mathcal{D}^{0^{-1}}$ where $\mathcal{K}_{\mu \nu}^{0}$ is the electromagnetic response function for non interacting electrons [2]. For $q<2 k_{F}$ and $\omega \ll k_{F} q / m, \mathcal{K}_{00}^{0} \simeq m / 2 \pi$ and $\mathcal{K}_{11}^{0} \simeq-\chi_{\mathrm{d}} q^{2}-k_{F}|\omega| / 4 \pi q$, where $\chi_{d}=(12 \pi m)^{-1}$ is the Landau diamagnetic susceptibility.

Within the RPA the screened density-current interaction in the static limit is

$$
V_{10}^{R P A}\left(\mathbf{k}, \mathbf{k}^{\prime}, \omega=0\right)=\frac{V_{10}^{0}\left(\mathbf{k}, \mathbf{k}^{\prime}\right)}{2 \pi m \tilde{\phi}^{2} \tilde{\chi}(q)}
$$

where $\tilde{\chi}(q)=v(q) /(2 \pi \tilde{\phi})^{2}+\left(1+6 / \tilde{\phi}^{2}\right) /(12 \pi m)$. Before proceeding it is interesting to study the dependence of the strength of this pairing interaction on the 'thickness' of the two dimensional electron gas. This can be done using the Stern-Howard potential for $v(q)$ corresponding to subband wave function $\xi(w)=A w \exp -b w / 2$ [22]. In order to address the well known failure of the various CS theories to renormalize the bare mass $m$ so that it is determined by $v(q)$, the dependence of the energy gap of the $\nu=1 / 3$ fractional quantum Hall state on the parameter $\beta=\left(b l_{0}\right)^{-1}$, computed variationally in [23, has been used. The results of these calculations are well fit by the simple expression $\Delta_{1 / 3}(\beta) \simeq 0.1 /(1+0.7 \beta) e^{2} / l_{0}$ which, using the relation $\Delta_{1 / 3}(\beta)=e B_{e f f} / m c=1 / 3 m l_{0}^{2}$, gives the $\beta$ dependent effective mass, $m(\beta) \simeq(10+7 \beta) /\left(3 e^{2} l_{0}\right)$. Figure 11 shows the dependence of $\lambda_{10}(0)$ on the thickness parameter $\beta$, computed using (5) with $l=1$ and $\tilde{\phi}=2$, both with and without this mass renormalization. The effect of including the screened density-density interaction $V_{00}(\mathbf{q}, \omega=$ $0)=\left(v_{q}+(2 \pi \tilde{\phi})^{2} /(12 \pi m)\right) /\left(2 \pi m \tilde{\phi}^{2} \tilde{\chi}(q)\right)$ through the corresponding coupling constant $\lambda_{00}(0)$ is also shown. In all cases the effective $p$-wave pairing interaction grows with thickness.

So far the current-current interaction between CS fermions mediated by the transverse CS gauge fluctuations has been ignored. In the Cooper channel this interaction is

$$
V_{11}^{R P A}\left(\mathbf{k}, \mathbf{k}^{\prime}, \omega_{n}\right)=\left(\frac{\mathbf{k} \times \hat{\mathbf{q}}}{m}\right)^{2} \mathcal{D}_{11}\left(q, \omega_{n}\right) \simeq\left(\frac{\mathbf{k} \times \hat{\mathbf{q}}}{m}\right)^{2} \frac{1}{\tilde{\chi}(q) q^{2}+\left(k_{F} / 2 \pi\right)\left|\omega_{n}\right| / q}
$$

It is convenient to first consider the case of short-range electron-electron interactions. Taking $v(q) \simeq v(0)$ and evaluating (5) gives

$$
\lambda_{11}\left(\omega_{n}\right) \sim-\frac{1}{m}\left(\frac{k_{F}}{\tilde{\chi}(0)}\right)^{2 / 3}\left|\omega_{n}\right|^{-1 / 3}
$$


Because the divergence of $\lambda_{11}(\omega)$ as $\omega \rightarrow 0$ comes from small $q$ scattering of Cooper pairs it is independent of $l$. Note that this singularity is repulsive and hence pair breaking in all angular momentum channels.

The effect of this singularity on the pairing instability of the CFL can be seen by considering the following simplified $T=0$ BCS gap equation,

$$
\Delta(\omega)=\lambda \int_{-\omega_{0}}^{\omega_{0}} d \omega^{\prime} \frac{\Delta\left(\omega^{\prime}\right)}{2 \sqrt{\omega^{\prime 2}+\left|\Delta\left(\omega^{\prime}\right)\right|^{2}}}-\gamma \int_{-\infty}^{\infty} d \omega^{\prime} \frac{\Delta\left(\omega^{\prime}\right)}{2 \sqrt{\omega^{\prime 2}+\left|\Delta\left(\omega^{\prime}\right)\right|^{2}}}\left(\frac{\omega_{0}}{\left|\omega-\omega^{\prime}\right|}\right)^{1 / 3} .
$$

Here $\lambda$ and $\gamma$ are dimensionless coupling constants characterizing a nonsingular attractive interaction and a singular repulsive interaction and $\omega_{0}$ is a characteristic energy scale (presumably of order $\left.e^{2} / l_{0}\right)$. Assuming that the $\omega$ dependence of $\Delta$ is weak, taking $\omega=0$, and performing the integrals yields

$$
1 \simeq \lambda \log \frac{\omega_{0}}{|\Delta|}-C \gamma\left(\frac{\omega_{0}}{|\Delta|}\right)^{1 / 3}
$$

where $C \simeq 4.2$. For small $\Delta$, the second term which prevents pairing dominates. This term is present in all angular momentum channels suggesting that the CFL may be immune from the GWW instability, or, for that matter, any Kohn-Luttinger-type instability [24], at least for short-range electron-electron interactions.

This analysis leaves out both self-energy effects and the self-consistent modification of the CS gauge field propagator, both of which may be important, particularly for the more physically relevant Coulomb interaction case for which the CS gauge fluctuations lead only to logarithmic singularities. An alternative approach which in principle includes these effects was introduced by Ubbens and Lee [25] in the context of the $U(1)$ gauge-theory description of the $t-J$ model. In this approach the free energy or, at $T=0$, the condensation energy, is computed directly within the RPA as a function of the gap parameter.

To apply the Ubbens-Lee analysis to the present problem it is necessary to 'probe' the CFL by introducing an $l$-wave pairing interaction by hand. This interaction is taken to be of the usual separable form,

$$
\mathcal{S}_{B C S}=\frac{V_{0}}{\mathcal{A} \beta} \sum_{m, n, n^{\prime}} \sum_{\mathbf{k}, \mathbf{k}^{\prime}} \bar{\gamma}_{\mathbf{k}} \gamma_{\mathbf{k}^{\prime}} \bar{\psi}\left(\mathbf{k}, \Omega_{n}+\omega_{m}\right) \bar{\psi}\left(-\mathbf{k},-\Omega_{n}\right) \psi\left(\mathbf{k}^{\prime}, \Omega_{n^{\prime}}+\omega_{m}\right) \psi\left(-\mathbf{k}^{\prime},-\Omega_{n^{\prime}}\right),
$$


where $\mathcal{A}$ is the area of the system, $\Omega_{n}=(2 n+1) \pi / \beta$ is a fermionic Matsubara frequency, $\gamma_{k}=\Theta\left(\omega_{0}+\epsilon_{k}\right) \Theta\left(\omega_{0}-\epsilon_{k}\right) \exp \left(i \theta_{k}\right), \epsilon_{k}=\left(k^{2}-k_{F}^{2}\right) / 2 m$ and $\theta_{k}=\arctan k_{y} / k_{x}$. The Hubbard-Stratonovich decomposition of the BCS interaction is accomplished by adding the term $\mathcal{S}_{H S}=\sum_{m} \bar{c}\left(\omega_{m}\right) c\left(\omega_{m}\right)$ to the action where $c\left(\omega_{m}\right)=\sqrt{\mathcal{A} \beta / V_{0}} \Delta\left(\omega_{n}\right)+$ $\sqrt{V_{0} / \mathcal{A} \beta} \sum_{n} \sum_{\mathbf{k}} \gamma_{\mathbf{k}} \psi\left(\mathbf{k}, \Omega_{n}+\omega_{m}\right) \psi\left(-\mathbf{k},-\Omega_{n}\right)$ [26]. The CS fermion fields can then be integrated out and, within the static approximation, $\Delta\left(\omega_{n}\right)=\Delta_{0} \delta_{n, 0}$, the resulting effective action can be expanded to second order in the CS gauge fields. Integrating out these fields and taking the $T=0$ limit then yields the RPA expression for the condensation energy per unit area,

$$
E\left(\Delta_{0}\right)=\frac{\left|\Delta_{0}\right|^{2}}{V_{0}}-\frac{m}{2 \pi} \int_{-\omega_{0}}^{\omega_{0}}\left(\sqrt{\epsilon^{2}+\left|\Delta_{0}\right|^{2}}-|\epsilon|\right) d \epsilon+E_{C S}\left(\Delta_{0}\right)-E_{C S}(0)
$$

where

$$
E_{C S}\left(\Delta_{0}\right)=\frac{1}{2} \int_{-\infty}^{\infty} \frac{d \omega}{2 \pi} \int \frac{d^{2} q}{(2 \pi)^{2}} \ln \operatorname{det} \mathcal{D}^{-1}\left(q, \omega ; \Delta_{0}\right)
$$

Here $\mathcal{D}_{\mu \nu}\left(\Delta_{0}\right)$ is the CS gauge field propagator obtained from the equation $\mathcal{D}^{-1}\left(\Delta_{0}\right)=$ $\mathcal{K}\left(\Delta_{0}\right)+\mathcal{D}^{0^{-1}}$ where now $\mathcal{K}_{\mu \nu}\left(\Delta_{0}\right)$ is the electromagnetic response function of the paired state calculated for fermions described by the Hamiltonian $H=\sum_{\mathbf{k}}\left(\epsilon_{k} \psi_{\mathbf{k}}^{\dagger} \psi_{\mathbf{k}}+\left[\Delta_{0} \sum_{\mathbf{k}} \gamma_{\mathbf{k}} \psi_{\mathbf{k}}^{\dagger} \psi_{-\mathbf{k}}^{\dagger}+\right.\right.$ H.c.]). To analyze (14) it is useful to analytically continue to the real frequency axis, setting $D_{\mu \nu}\left(q, \nu=i\left|\omega_{n}\right|\right)=\mathcal{D}_{\mu \nu}(q, \omega)$ and $K_{\mu \nu}(q, \nu=|\omega|)=\mathcal{K}_{\mu \nu}(q, \omega)$. The usual deformation of the imaginary frequency integration in (14) around the branch cut of the logarithm on the real axis then gives [2,25]

$$
E_{C S}\left(\Delta_{0}\right)=\int_{0}^{\infty} \frac{d \nu}{2 \pi} \int \frac{d^{2} q}{(2 \pi)^{2}} \tan ^{-1} \frac{\operatorname{Im} \operatorname{det} D^{-1}\left(q, \nu ; \Delta_{0}\right)}{\operatorname{Re} \operatorname{det} D^{-1}\left(q, \nu ; \Delta_{0}\right)}
$$

In the paired state at $T=0$ there is no damping for frequencies $\nu \leq 2\left|\Delta_{0}\right|$ which implies that $\operatorname{Im} K_{00}\left(\Delta_{0}\right)=\operatorname{Im} K_{11}\left(\Delta_{0}\right)=\operatorname{Re} K_{01}\left(\Delta_{0}\right)=\operatorname{Re} K_{10}\left(\Delta_{0}\right)=0$ and thus $\operatorname{Im} \operatorname{det} D^{-1}\left(\Delta_{0}\right)=$ 0 for $\nu \leq 2\left|\Delta_{0}\right|$. Following [25] $E_{C S}\left(\Delta_{0}\right)-E_{C S}(0)$ can then be estimated by calculating the contributions of these frequencies to $-E_{C S}(0)$ which are lost in the paired state [27]. For the case of short-range electron-electron interactions, taking $v(q) \simeq v(0)$, this approximation gives 


$$
E_{C S}\left(\Delta_{0}\right)-E_{C S}(0) \simeq \int_{0}^{2\left|\Delta_{0}\right|} \frac{d \nu}{2 \pi} \int \frac{d^{2} q}{(2 \pi)^{2}} \tan ^{-1} \frac{k_{F}}{2 \pi \tilde{\chi}(0)} \frac{\nu}{q^{3}} \sim\left(\frac{k_{F}}{\tilde{\chi}(0)}\right)^{2 / 3}\left|\Delta_{0}\right|^{5 / 3}
$$

For small $\Delta_{0}$ this term, which is consistent with the singular term appearing in (11), will always dominate the condensation energy and prevent any continuous zero temperature pairing transition. Thus for short-range electron-electron interactions any pairing transition of the CFL will necessarily involve a discontinuous jump in $\Delta_{0}$ and so be first order. As pointed out in [25], the appearance in $E\left(\Delta_{0}\right)$ of a nonanalytic term in $\left|\Delta_{0}\right|^{2}$ due to finite frequency gauge fluctuations is a quantum version of the fluctuation driven first-order transition discussed by Halperin, Lubensky and Ma [28]. A similar effect, in which a continuous quantum Hall/insulator transition is driven first-order by a fluctuating CS gauge field, has been studied by Pryadko and Zhang [29].

If the same approximation is applied to the Coulomb interaction case it is necessary to cut off the momentum integration at $2 k_{F}$ and the result is

$$
E_{C S}\left(\Delta_{0}\right)-E_{C S}(0) \simeq \int_{0}^{2\left|\Delta_{0}\right|} \frac{d \nu}{2 \pi} \int_{0}^{2 k_{F}} \frac{q d q}{2 \pi} \tan ^{-1} \frac{k_{F} \tilde{\phi}^{2}}{e^{2}} \frac{\nu}{q^{2}} \simeq \frac{m_{c}}{2 \pi}\left|\Delta_{0}\right|^{2} \ln \frac{2 k_{F} e^{2}}{\tilde{\phi}^{2}\left|\Delta_{0}\right|}
$$

where $m_{c}=k_{F} \tilde{\phi}^{2} / 2 \pi e^{2}$. For small $\Delta_{0}$ the condensation energy is then $E\left(\Delta_{0}\right) \sim\left|\Delta_{0}\right|^{2} / V_{0}-$ $\left(\left(m-m_{c}\right) / 2 \pi\right)\left|\Delta_{0}\right|^{2} \ln \left(\omega_{0} /\left|\Delta_{0}\right|\right)$. If $m-m_{c}<0$ pair-breaking dominates and any $T=0$ pairing transition will be first order. Alternatively, if $m-m_{c}>0$ a continuous transition is possible and the CFL is unstable for arbitrarily weak $V_{0}$. It is interesting to note that according to the mass renormalization scheme discussed above $m(\beta)-m_{c}>0$ for $\tilde{\phi}=2$ suggesting that for $\nu=1 / 2$ the CFL is unstable for Coulomb interactions, though it is unclear to what extent this result can be trusted.

Because the CS contribution to the condensation energy is due to low-energy, longwavelength fluctuations, it is unaffected by any softening of the short-range part of the electron-electron interaction. It follows that as the thickness parameter $\beta$ increases both the effective mass $m$ and $\lambda_{10}$, and hence the tendency towards pairing, increase while the pairbreaking effects are essentially unchanged. Thus the CFL becomes less stable with increasing thickness, consistent with Morf's interpretation of the $\nu=5 / 2$ tilted field experiments 10 
as well as numerical studies of the half-filled Landau level [7,10,13,30].

To summarize, the CS gauge fluctuations in the CFL description of the half-filled Landau level are strongly pair breaking in all angular momentum channels and so provide a hostile environment for the formation of Cooper pairs. For short-range electron-electron interactions these fluctuations are sufficiently strong to drive any $T=0$ pairing transition of the CFL first-order. For Coulomb interactions these fluctuations are weaker and, depending on details, a continuous transition may be possible. To the extent that more recent formulations of the CFL contain both a pairing interaction [15] and a $U(1)$ gauge field [18 20] the conclusions of this paper should still be relevant.

Useful discussions with V. Melik-Alaverdian are acknowledged. This work was supported by DOE grant No. DE-FG02-97ER45639 and the Alfred P. Sloan Foundation. 


\section{REFERENCES}

[1] H.W. Jiang et al., Phys. Rev. B 40, 12013 (1989); R.L. Willett et al., Phys. Rev. Lett. 65, 112 (1990); R.R. Du et al., ibid 70, 2944 (1992); W. Kang et al., ibid. 71, 3850 (1993); V.J. Goldman et al., ibid. 72, 2065 (1994); J.H. Smet et al., ibid. 77, 2272 (1996); Phys. Rev. B 56, 3606 (1997).

[2] B.I. Halperin, P.A. Lee, and N. Read, Phys. Rev. B 47, 7312 (1993).

[3] J.K. Jain, Phys. Rev. Lett. 63 (1989) 199; Phys. Rev. B 40, 8079 (1989); ibid. 41, 7653 (1990).

[4] V. Kalmeyer and S.C. Zhang, Phys. Rev. B, 46, 9889 (1992).

[5] A. Lopez and E. Fradkin, Phys. Rev. B 44, 5246 (1991).

[6] S.C. Zhang, H. Hanson, and S. Kivelson, Phys. Rev. Lett. 62, 82 (1989); 62, 980 (E) (1989).

[7] M. Greiter, X.G. Wen, and F. Wilczek, Phys. Rev. Lett. 66, 3205 (1991); Nucl. Phys. B 374, 567 (1992).

[8] G. Moore and N. Read, Nucl. Phys. B 360, 362 (1991).

[9] R.L. Willett et al., Phys. Rev. Lett. 59, 1776 (1987).

[10] R. Morf, Phys. Rev. Lett. 80, 1505 (1998).

[11] J.P. Eisenstein et al., Phys. Rev. Lett. 61, 997 (1988).

[12] For a discussion of this effect see T. Chakraborty and P. Pietiläinen, Phys. Rev. B 39, 7971 (1989).

[13] E.H. Rezayi and F.D.M. Haldane, Bull. Am. Phys. Soc. 43, 655 (1998).

[14] N. Read, Semicond. Sci. Technol. 9, 1859 (1994).

[15] G. Baskaran, Physica B 212, 320 (1995). 
[16] R. Shankar and G. Murthy, Phys. Rev. Lett. 79, 4437 (1997); G. Murthy and R. Shankar, cond-mat/9709233; cond-mat/9806380.

[17] V. Pasquier and F.D.M. Haldane, cond-mat/9712169.

[18] D.-H. Lee, Phys. Rev. Lett. 80, 4745 (1998).

[19] N. Read, cond-mat/9804294.

[20] B.I. Halperin and A. Stern, Phys. Rev. Lett. 80, 5457 (1998); G. Murthy and R. Shankar, ibid. 80, 5458 (1998).

[21] It is an artifact of the Fermi surface approximation that $\lambda_{10}^{0}$ is independent of $l$. In [7] the full BCS gap equation was solved and the leading instability found in the $l=1$ channel.

[22] F. Stern and W.E. Howard, Phys. Rev. 163, 816 (1967); T. Ando, A.B. Fowler, and F. Stern, Rev. Mod. Phys. 54, 437 (1982).

[23] V. Melik-Alaverdian and N.E. Bonesteel, Phys. Rev. B 52, R17032 (1995).

[24] W. Kohn and J.M. Luttinger, Phys. Rev. Lett. 15, 524 (1965).

[25] M.U. Ubbens and P.A. Lee, Phys. Rev. B 49, 6853 (1994).

[26] See, for example, V.N. Popov Functional Integrals and Collective Excitations (Cambridge University Press, Cambridge, 1987).

[27] Though this approximation ignores the modification of $K$ for $\nu>2\left|\Delta_{0}\right|$, it is assumed here that the pair-breaking effects due to low-frequency fluctuations are adequately captured.

[28] B.I. Halperin, T.C. Lubensky, and S.K. Ma, Phys. Rev. Lett. 32, 292 (1974).

[29] L. Pryadko and S.C. Zhang, Phys. Rev. Lett. 73, 3282 (1994).

[30] K. Park, V. Melik-Alaverdian, N.E. Bonesteel, and J. Jain, cond-mat/9806271. 


\section{FIGURES}

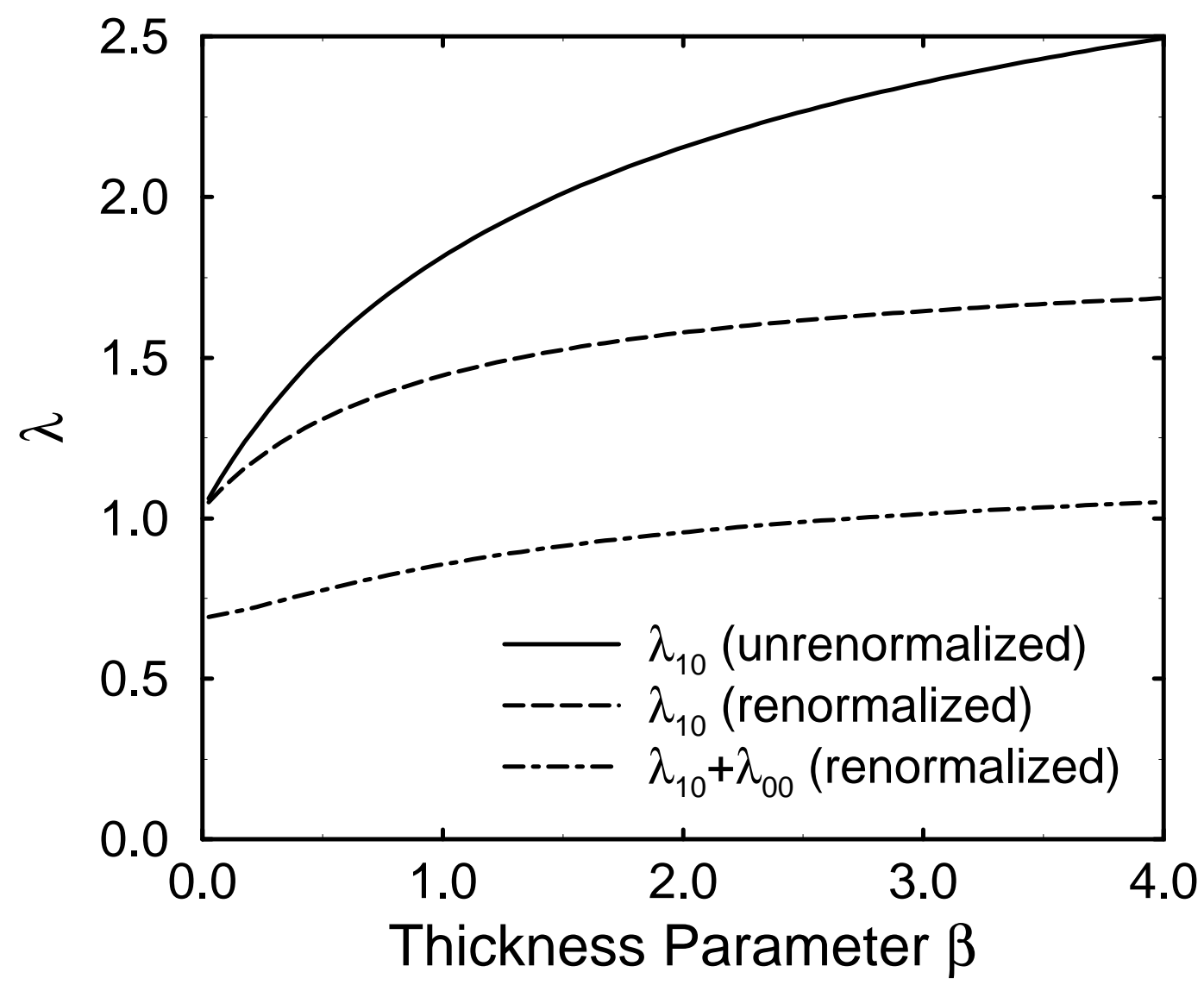

FIG. 1. Coupling constant characterizing the strength of the $p$-wave pairing interaction vs. thickness parameter $\beta$. Results are given with (solid) and without (dashed) the mass renormalization discussed in the text, as well as with both the mass renormalization and the screened Coulomb repulsion (dot-dashed). In all cases the interaction grows stronger as the thickness is increased. 